\title{
Gaussian time-dependent variational principle for the finite-temperature anharmonic lattice dynamics
}

\author{
Jae-Mo Lihm (1)* and Cheol-Hwan Park $\oplus^{\dagger}$ \\ Center for Correlated Electron Systems, Institute for Basic Science, Seoul 08826, Korea; \\ Department of Physics and Astronomy, Seoul National University, Seoul 08826, Korea; \\ and Center for Theoretical Physics, Seoul National University, Seoul 08826, Korea
}

(Received 3 December 2020; revised 21 May 2021; accepted 7 July 2021; published 23 July 2021)

\begin{abstract}
The anharmonic lattice is a representative example of an interacting, bosonic, many-body system. The selfconsistent harmonic approximation has proven versatile for the study of the equilibrium properties of anharmonic lattices. However, the study of dynamical properties therein resorts to an ansatz, whose validity has not yet been theoretically proven. Here we apply the time-dependent variational principle, a recently emerging useful tool for studying the dynamic properties of interacting many-body systems, to the anharmonic lattice Hamiltonian at finite temperature using the Gaussian states as the variational manifold. We derive an analytic formula for the position-position correlation function and the phonon self-energy, proving the dynamical ansatz of the self-consistent harmonic approximation. We establish a fruitful connection between time-dependent variational principle and the anharmonic lattice Hamiltonian, providing insights in both fields. Our work expands the range of applicability of the time-dependent variational principle to first-principles lattice Hamiltonians and lays the groundwork for the study of dynamical properties of the anharmonic lattice using a fully variational framework.
\end{abstract}

DOI: 10.1103/PhysRevResearch.3.L032017

Introduction. Variational methods form the basis of our understanding of quantum-mechanical many-body systems. In a variational method, the wave functions or density matrices of a system are parametrized by a set of parameters whose size is much smaller than the dimension of the Hilbert space. Static and time-dependent [1-3] variational methods are being actively used to study interacting many-body model Hamiltonians [4-13].

The anharmonic lattice is a representative example of an interacting bosonic many-body system in materials science. The self-consistent harmonic approximation (SCHA) is a variational method for approximately finding the ground or thermal equilibrium state of an anharmonic lattice Hamiltonian $[14,15]$. Recently, a stochastic implementation of SCHA [16-19] was developed and attracted considerable attention. SCHA has been successfully applied to study structural phase transitions [18-21], superconductivity [16,22-25], and charge density waves [26-31], as well as to the dynamical properties such as the phonon spectral function [20,21,32-34] and infrared and Raman spectra [35].

However, SCHA is limited in that one needs to resort to a specific ansatz to study the dynamical properties. It is known that the SCHA ansatz for the position-position

\footnotetext{
*jaemo.lihm@gmail.com

†cheolhwan@snu.ac.kr
}

Published by the American Physical Society under the terms of the Creative Commons Attribution 4.0 International license. Further distribution of this work must maintain attribution to the author(s) and the published article's title, journal citation, and DOI.
Green function is correct in the static limit of zero frequency and the perturbative limit of weak anharmonicity [18]. However, the validity of the SCHA ansatz in the nonperturbative and dynamic regime [20,21,33,35], where the dynamical theory is most necessary, has not been theoretically justified.

In this Letter, we solve this important problem by applying the time-dependent variational principle (TDVP) with Gaussian variational states $[7,13,36,37]$ to the anharmonic lattice Hamiltonian at finite temperature. Gaussian TDVP expands the static variational states of SCHA to states with nonzero momenta. We use the linearized time evolution to derive the position-position correlation function and prove the SCHA dynamical ansatz. We illustrate that the Gaussian TDVP is successful in describing the dynamics because it includes the two-phonon states as true dynamical excitations. Our work connects the TDVP theory, whose application was mostly focused on model Hamiltonians for cold atoms, with anharmonic lattice dynamics and the SCHA method. Such connection gives fruitful results on both sides. In TDVP theory, the linearized time evolution and the projected Hamiltonian method $[4,6,9]$ are two different ways to compute the excitation spectrum, whose superiority over the other varies across systems $[4,7,13]$. We use the anharmonic lattice model to show that only the linearized time evolution gives correct excitation energies in the perturbative limit. On the SCHA side, we illustrate ways to systematically expand the SCHA theory by leveraging recent developments of non-Gaussian TDVP $[6,11,12]$.

Self-consistent harmonic approximation. We briefly review the key results of SCHA. Within the adiabatic Born-Oppenheimer approximation, the anharmonic lattice 
Hamiltonian is

$$
\hat{H}=\sum_{a=1}^{N} \frac{\hat{\tilde{p}}_{a}^{2}}{2 M_{a}}+\widetilde{V}\left(\hat{\widetilde{r}}_{1}, \ldots, \hat{\widetilde{r}}_{N}\right) .
$$

Here, $a$ is the combined index for atoms and Cartesian directions; $N=N_{\text {atm }} \times d$ with $N_{\text {atm }}$ and $d$ the numbers of the atoms and the spatial dimensions, respectively; $M_{a}$ the atomic mass; $\hat{\tilde{r}}_{a}$ and $\hat{\widetilde{p}}_{a}$ the position and momentum operators; and $\widetilde{V}$ the Born-Oppenheimer potential energy. We set $\hbar=1$.

In SCHA, the true thermal equilibrium state of the anharmonic Hamiltonian is approximated by that of a harmonic Hamiltonian $\hat{H}^{(\mathrm{H})}$ :

$$
\hat{H}^{(\mathrm{H})}=\sum_{a=1}^{N} \frac{\hat{\widetilde{p}}_{a}^{2}}{2 M_{a}}+\widetilde{V}^{(\mathrm{H})}(\hat{\tilde{\mathbf{r}}}) .
$$

Since we study the dynamics around the SCHA equilibrium, we assume that the optimal harmonic potential $\widetilde{V}^{(\mathrm{H})}$ is already found. The SCHA density matrix is

$$
\hat{\rho}_{0}=e^{-\beta \hat{H}^{(\mathrm{H})}} / \operatorname{Tr} e^{-\beta \hat{H}^{(\mathrm{H})}},
$$

where $\beta=1 / k_{\mathrm{B}} T$ is the inverse temperature. For later use, we define $\langle\hat{A}\rangle_{0} \equiv \operatorname{Tr}\left(\hat{\rho}_{0} \hat{A}\right)$.

Hereafter, we use the normal-mode representation, where the SCHA harmonic Hamiltonian becomes

$$
\hat{H}^{(\mathrm{H})}=\sum_{m=1}^{N} \frac{\omega_{m}}{2}\left(\hat{p}_{m}^{2}+\hat{r}_{m}^{2}\right),
$$

with $\omega_{m}$ the eigenvalue of the SCHA dynamical matrix, and $\hat{r}_{m}$ and $\hat{p}_{m}$ the normal-mode position and momentum operators. The anharmonic Hamiltonian [Eq. (1)] can be written as

$$
\hat{H}=\sum_{m=1}^{N} \frac{\omega_{m}}{2} \hat{p}_{m}^{2}+V(\hat{\mathbf{r}}),
$$

with $V(\hat{\mathbf{r}})=\widetilde{V}(\hat{\mathbf{r}})$ the potential energy in the normal-mode representation.

In the normal-mode representation, the SCHA selfconsistency equations [18] become

$$
\left\langle\frac{\partial \hat{V}}{\partial r_{m}}\right\rangle_{0}=0, \quad\left\langle\frac{\partial^{2} \hat{V}}{\partial r_{m} \partial r_{n}}\right\rangle_{0}=\omega_{m} \delta_{m, n} .
$$

Also, since $\hat{\rho}_{0}$ is a thermal state, we find

$$
\left\langle\hat{p}_{m}\right\rangle_{0}=0, \quad\left\langle\hat{p}_{m} \hat{p}_{n}\right\rangle_{0}=\left(n_{m}+\frac{1}{2}\right) \delta_{m, n},
$$

with $n_{m}=1 /\left(e^{\beta \omega_{m}}-1\right)$ the occupation number.

Gaussian time-dependent variational principle. Next we discuss the general principles of Gaussian TDVP for a multimode bosonic system at finite temperature. We use the set of states obtained by applying a Gaussian unitary transformation $\hat{U}(\mathbf{x})$ to the SCHA density matrix as the variational manifold:

$$
\hat{\rho}(\mathbf{x})=\hat{U}(\mathbf{x}) \hat{\rho}_{0} \hat{U}^{\dagger}(\mathbf{x}) \text {. }
$$

Here, $\mathbf{x}$ is a real-valued vector that encodes all the variational parameters. We parametrize the Gaussian transformation as

$$
\hat{U}(\mathbf{x})=\hat{D}(\boldsymbol{\alpha}) \hat{S}(\boldsymbol{\beta}, \boldsymbol{\gamma}),
$$

where $\hat{D}$ and $\hat{S}$ are the displacement and squeezing operators, respectively:

$$
\begin{array}{r}
\hat{D}(\boldsymbol{\alpha})=\exp \left(\frac{1}{\sqrt{2}} \sum_{m}\left(\alpha_{m} \hat{a}_{m}^{\dagger}-\alpha_{m}^{*} \hat{a}_{m}\right)\right), \\
\hat{S}(\boldsymbol{\beta}, \boldsymbol{\gamma})=\exp \left[\sum_{\substack{m, n \\
m \leqslant n}} b_{m n}\left(\beta_{m n} \hat{a}_{m}^{\dagger} \hat{a}_{n}^{\dagger}-\beta_{m n}^{*} \hat{a}_{m} \hat{a}_{n}\right)\right. \\
\left.+\sum_{\substack{m, n \\
m<n}} c_{m n}\left(\gamma_{m n}^{*} \hat{a}_{m}^{\dagger} \hat{a}_{n}-\gamma_{m n} \hat{a}_{n}^{\dagger} \hat{a}_{m}\right)\right],
\end{array}
$$

where

$$
\begin{gathered}
b_{m n} \equiv \begin{cases}1 / \sqrt{4\left(n_{m}+n_{n}+1\right)} & \text { if } m=n \\
1 / \sqrt{2\left(n_{m}+n_{n}+1\right)} & \text { if } m \neq n\end{cases} \\
c_{m n} \equiv 1 / \sqrt{2\left(n_{m}-n_{n}\right)} .
\end{gathered}
$$

The variational parameters $\alpha_{m}, \beta_{m n}$, and $\gamma_{m n}$ are complex numbers. The parameter $\beta_{m n}\left(\gamma_{m n}\right)$ is defined only for $m \leqslant n$ $(m<n)$. Here, we assume for simplicity that $\omega_{m}$ 's are nondegenerate and satisfy $\omega_{1}<\omega_{2}<\cdots<\omega_{N}$. The total number of complex variational parameters is $N^{2}+N$. In the linear response regime, degeneracy does not pose any theoretical difficulty: if modes $m$ and $n$ are degenerate, one just needs to exclude $\gamma_{m n}$ from the set of variational parameters. This exclusion is done because the infinitesimal transformation parametrized by $\gamma_{m n}$ does not change $\hat{\rho}_{0}$ [38].

Each group of parameters describes a different type of excitation. Parameters $\boldsymbol{\alpha}, \boldsymbol{\beta}$, and $\boldsymbol{\gamma}$ correspond to one-phonon excitations, two-phonon excitations with two creations or two annihilations of phonons, and two-phonon excitations with one creation and one annihilation, respectively.

The imaginary parts of the parameters generate dynamics. For example, Im $\alpha$ generates a finite atomic momentum through the displacement operator. The SCHA theory does not contain these imaginary parameters because the variational states are limited to the thermal state of a harmonic Hamiltonian. In contrast, Gaussian TDVP, which allows both the real and imaginary parts of the variational parameters to vary, naturally allows one to study the dynamics.

We define $\mathbf{x}$, the vector of variational parameters, as

$$
\mathbf{x}=(\operatorname{Re} \boldsymbol{\alpha} \operatorname{Im} \boldsymbol{\alpha} \operatorname{Re} \boldsymbol{\beta} \operatorname{Im} \boldsymbol{\beta} \operatorname{Re} \boldsymbol{\gamma} \operatorname{Im} \boldsymbol{\gamma})^{\top} .
$$

Since $\hat{\rho}(\mathbf{x}=0)=\hat{\rho}_{0}$ is the variational solution that minimizes the SCHA free energy, $\mathbf{x}=0$ is a stationary point of the variational time evolution [38].

To apply TDVP to mixed states, we map the variational density matrices to wave functions by purification [11,39]. For each physical state in the number basis, we add an auxiliary state so that the purified wave function becomes

$$
|\Psi(\mathbf{x})\rangle=\left[\hat{U}(\mathbf{x}) \sqrt{\hat{\rho}_{0}} \otimes \mathbb{1}\right]\left|\Phi^{+}\right\rangle,
$$

where $\otimes$ denotes a tensor product and $\left|\Phi^{+}\right\rangle$is a maximally entangled state [39] between the physical and the auxiliary modes [see Eq. (S15) and related discussions]. For the purified wave function, the expectation value of a physical operator $\hat{A}_{0}$ is

$$
A(\mathbf{x}) \equiv\left\langle\Psi(\mathbf{x})\left|\hat{A}_{0} \otimes \mathbb{1}\right| \Psi(\mathbf{x})\right\rangle=\langle\hat{A}(\mathbf{x})\rangle_{0},
$$


where

$$
\hat{A}(\mathbf{x})=\hat{U}^{\dagger}(\mathbf{x}) \hat{A}_{0} \hat{U}(\mathbf{x}) .
$$

The variational time evolution is obtained by projecting the true dynamics of the wave function to the tangent space of the variational manifold. The tangent space is spanned by the tangent vectors, which at $\mathbf{x}=0$ are

$$
\left|V_{\mu}\right\rangle=\left[\left(\partial_{\mu} \hat{U}\right) \sqrt{\hat{\rho}_{0}} \otimes \mathbb{1}\right]\left|\Phi^{+}\right\rangle .
$$

Using the variational linear response theory [13,38], one can show that the retarded correlation function $G_{A B}^{(\mathrm{R})}(\omega)$ between operators $\hat{A}$ and $\hat{B}$ is

$$
G_{A B}^{(\mathrm{R})}(\omega)=\lim _{\eta \rightarrow 0^{+}}-i\left(\partial_{\mu} B\right) \mathcal{G}^{\mu}{ }_{\nu}(\omega+i \eta)\left(\Omega^{\nu \rho} \partial_{\rho} A\right) .
$$

Here, the matrix Green function $\mathcal{G}(z)$ is defined as

$$
(z-i \mathbf{K}) \mathcal{G}(z)=\mathbb{1},
$$

where $\mathbf{K}$ is the linearized time-evolution generator defined as

$$
K^{\mu}{ }_{\nu}=-\Omega^{\mu \rho} \partial_{\rho} \partial_{\nu} E,
$$

with $E(\mathbf{x})=\operatorname{Tr}[\hat{\rho}(\mathbf{x}) \hat{H}]$. The symplectic form $\boldsymbol{\Omega}$ is defined by

$$
\Omega^{\mu \rho} \operatorname{Im}\left\langle V_{\rho} \mid V_{\nu}\right\rangle=\frac{1}{2} \delta^{\mu}{ }_{\nu} .
$$

By computing $\mathbf{K}$ and the corresponding matrix Green function $\mathcal{G}(z)$, one can find the physical correlation function $G_{A B}^{(\mathrm{R})}(\omega)$ using Eq. (19).

Anharmonic lattice dynamics. Now, we study the dynamical properties of the anharmonic lattice Hamiltonian using Gaussian TDVP. First, the symplectic form is [38]

$$
\boldsymbol{\Omega}=\left(\begin{array}{cc}
0 & -\mathbb{1} \\
\mathbb{1} & 0
\end{array}\right) \oplus\left(\begin{array}{cc}
0 & -\mathbb{1} \\
\mathbb{1} & 0
\end{array}\right) \oplus\left(\begin{array}{cc}
0 & -\mathbb{1} \\
\mathbb{1} & 0
\end{array}\right),
$$

with $\oplus$ the direct sum.

The three matrices correspond to the subspace spanned by the tangent vectors for the variation of $\boldsymbol{\alpha}, \boldsymbol{\beta}$, and $\boldsymbol{\gamma}$, respectively. In each matrix the bases for the first (second) block of rows and columns are the tangent vectors for the real (imaginary) parts of the parameters.

For later use, we define $\mathbf{P}_{1}, \mathbf{P}_{2+}$, and $\mathbf{P}_{2-}$ as the projection operators to the bases of each of the three matrices. The subscripts $1,2+$, and $2-$ indicate the nature of the tangent vectors: one-phonon excitations, two-phonon excitations with two creations or two annihilations, and two-phonon excitations with one creation and one annihilation. We also define the projection to the whole two-phonon sector: $\mathbf{P}_{2}=\mathbf{P}_{2+}+$ $\mathbf{P}_{2-}$.

Evaluating Eq. (21), we find that the time-evolution generator $\mathbf{K}$ is the sum of the noninteracting part, three-phonon interaction, and four-phonon interaction (see Sec. S4 C of the Supplemental Material [38]):

$$
i \mathbf{K}=\mathbf{H}^{(0)}+\mathbf{V}^{(3)}+\mathbf{V}^{(4)},
$$

where

$$
\mathbf{H}^{(0)}=\left(\begin{array}{cc}
0 & i \omega \\
-i \omega & 0
\end{array}\right) \oplus\left(\begin{array}{cc}
0 & i \omega_{+} \\
-i \omega_{+} & 0
\end{array}\right) \oplus\left(\begin{array}{cc}
0 & i \omega_{-} \\
-i \omega_{-} & 0
\end{array}\right),
$$

$$
\begin{aligned}
\mathbf{V}^{(3)} & =\left(\begin{array}{cccccc}
0 & 0 & 0 & 0 & 0 & 0 \\
0 & 0 & -i \boldsymbol{\Phi}^{(3)} B & 0 & -i \boldsymbol{\Phi}^{(3)} C & 0 \\
0 & 0 & 0 & 0 & 0 & 0 \\
-i B \boldsymbol{\Phi}^{(3)} & 0 & 0 & 0 & 0 & 0 \\
0 & 0 & 0 & 0 & 0 & 0 \\
-i C \boldsymbol{\Phi}^{(3)} & 0 & 0 & 0 & 0 & 0
\end{array}\right) \\
\mathbf{V}^{(4)} & =\left(\begin{array}{ll}
0 & 0 \\
0 & 0
\end{array}\right) \oplus\left(\begin{array}{cccc}
0 & 0 & 0 & 0 \\
-i B \boldsymbol{\Phi}^{(4)} B & 0 & -i B \boldsymbol{\Phi}^{(4)} C & 0 \\
0 & 0 & 0 & 0 \\
-i C \boldsymbol{\Phi}^{(4)} B & 0 & -i C \boldsymbol{\Phi}^{(4)} C & 0
\end{array}\right) .
\end{aligned}
$$

Here, we defined the diagonal matrices:

$$
\begin{gathered}
\boldsymbol{\omega}_{m, n}=\omega_{m} \delta_{m, n}, \\
{\left[\omega_{ \pm}\right]_{m n, p q}=\left(\omega_{m} \pm \omega_{n}\right) \delta_{m n, p q},} \\
B_{m n, p q}=b_{m n}\left(n_{m}+n_{n}+1\right) \delta_{m n, p q}, \\
C_{m n, p q}=-c_{m n}\left(n_{m}-n_{n}\right) \delta_{m n, p q} .
\end{gathered}
$$

The implicit summation over a pair of mode indices $m$ and $n$ implies the constraint $m \leqslant n$ unless otherwise noted. We also defined the anharmonicity tensor

$$
\Phi_{n_{1}, \cdots, n_{m}}^{(m)}=\left\langle\frac{\partial^{m} V}{\partial r_{n_{1}} \cdots \partial r_{n_{m}}}\right\rangle_{0} .
$$

The noninteracting part $\mathbf{H}^{(0)}$ describes the free evolution of one- and two-phonon excitations in the SCHA Hamiltonian. The three-phonon interaction $\mathbf{V}^{(3)}$ couples the one- and twophonon excitations. The four-phonon interaction $\mathbf{V}^{(4)}$ couples the two-phonon excitations to each other.

Finally, we study the linear response of the anharmonic lattice and compute the position-position correlation function. First, we define the noninteracting Green function $\mathcal{G}^{(0)}$ :

$$
\left(z-\mathbf{H}^{(0)}\right) \mathcal{G}^{(0)}(z)=\mathbb{1} .
$$

From Eq. (25), one finds

$$
\mathcal{G}^{(0)}(z)=\mathcal{G}_{1}^{(0)}(z) \oplus \mathcal{G}_{2+}^{(0)}(z) \oplus \mathcal{G}_{2-}^{(0)}(z),
$$

where

$$
\mathcal{G}_{1}^{(0)}(z)=\frac{1}{z^{2}-\omega^{2}}\left(\begin{array}{cc}
z & i \omega \\
-i \omega & z
\end{array}\right),
$$

and

$$
\mathcal{G}_{2 \pm}^{(0)}(z)=\frac{1}{z^{2}-\omega_{ \pm}^{2}}\left(\begin{array}{cc}
z & i \omega_{ \pm} \\
-i \omega_{ \pm} & z
\end{array}\right) .
$$

Next, we include the four-phonon interaction $\mathbf{V}^{(4)}$. We define the partially interacting Green function $\mathcal{G}^{(4)}(z)$ :

$$
\left(z-\mathbf{H}^{(0)}-\mathbf{V}^{(4)}\right) \mathcal{G}^{(4)}(z)=\mathbb{1} .
$$

Since the four-phonon interaction $\mathbf{V}^{(4)}$ does not act on the onephonon sector, we find

$$
\mathbf{P}_{1} \mathcal{G}^{(4)} \mathbf{P}_{1}=\mathcal{G}_{1}^{(0)} \oplus 0 .
$$


For the two-phonon sector, we obtain the Dyson equation

$$
\mathbf{P}_{2} \mathcal{G}^{(4)} \mathbf{P}_{2}=\mathbf{P}_{2} \mathcal{G}^{(0)} \mathbf{P}_{2}+\mathbf{P}_{2} \mathcal{G}^{(4)} \mathbf{V}^{(4)} \mathcal{G}^{(0)} \mathbf{P}_{2}
$$

Finally, we study the fully interacting Green function $\mathcal{G}(z)$ by including the three-phonon interaction $\mathbf{V}^{(3)}$. From the definitions of $\mathcal{G}$ and $\mathcal{G}^{(4)}$, we obtain the Dyson equation

$$
\mathbf{P}_{1} \mathcal{G} \mathbf{P}_{1}=\mathbf{P}_{1} \mathcal{G}^{(4)} \mathbf{P}_{1}+\mathbf{P}_{1} \mathcal{G}^{(4)} \mathbf{P}_{1} \mathbf{V}^{(3)} \mathbf{P}_{2} \mathcal{G}^{(4)} \mathbf{P}_{2} \mathbf{V}^{(3)} \mathbf{P}_{1} \mathcal{G} \mathbf{P}_{1}
$$

One can solve the Dyson equations [Eqs. $(39,40)]$ to find [38]

$$
\begin{aligned}
& \mathbf{P}_{1} \mathcal{G} \mathbf{P}_{1} \\
& =\mathcal{G}_{1}^{(0)}-\mathcal{G}_{1}^{(0)}\left(\begin{array}{cc}
0 & 0 \\
\sum_{s, s^{\prime}= \pm} \boldsymbol{\Phi}^{(3)} B_{s}\left[\mathcal{G}_{s s^{\prime}}^{(4)}\right]_{12} B_{s^{\prime}} \boldsymbol{\Phi}^{(3)} & 0
\end{array}\right) \mathbf{P}_{1} \mathcal{G} \mathbf{P}_{1} .
\end{aligned}
$$

Here, we defined $B_{+}=B$ and $B_{-}=C$. In Eq. (41), we omitted the direct sum of the zero matrix in the $\mathbf{P}_{2}$ subspace for brevity.

From Eqs. (S1, S2), one finds that the matrix elements for the position operator is nonzero only for the variation of $\operatorname{Re} \alpha$ :

$$
\partial_{\mu} \mathbf{r}=\left(\begin{array}{llllll}
\mathbb{1} & 0 & 0 & 0 & 0 & 0
\end{array}\right)^{\top} .
$$

Then, from Eqs. $(19,41)$, one can derive the Dyson equation for the interacting retarded position-position correlation function [38]:

$$
\mathbf{G}_{r r}^{(\mathrm{R})}=\mathbf{G}_{r r}^{(\mathrm{R} 0)}+\mathbf{G}_{r r}^{(\mathrm{R} 0)} \boldsymbol{\Pi}_{r r} \mathbf{G}_{r r}^{(\mathrm{R})} .
$$

The self-energy is

$$
\boldsymbol{\Pi}_{r r}(z)=\boldsymbol{\Phi}^{(3)} \mathbf{W}\left(\mathbb{1}-\boldsymbol{\Phi}^{(4)} \mathbf{W}\right)^{-1} \boldsymbol{\Phi}^{(3)},
$$

where $\mathbf{W}$ is a diagonal matrix defined as

$$
\mathbf{W}=\sum_{s= \pm} B_{s} \frac{\omega_{s}}{z^{2}-\omega_{s}^{2}} B_{s} .
$$

By recovering the mode indices and defining

$$
\begin{aligned}
\chi_{m n, p q}(z) \equiv & \frac{1}{2}\left[\frac{\left(\omega_{m}+\omega_{n}\right)\left(n_{m}+n_{n}+1\right)}{\left(\omega_{m}+\omega_{n}\right)^{2}-z^{2}}\right. \\
& \left.-\frac{\left(\omega_{m}-\omega_{n}\right)\left(n_{m}-n_{n}\right)}{\left(\omega_{m}-\omega_{n}\right)^{2}-z^{2}}\right] \delta_{m n, p q},
\end{aligned}
$$

one can rewrite Eq. (44) in a form identical to the SCHA dynamical ansatz [38]:

$$
\boldsymbol{\Pi}_{r r}(z)=\boldsymbol{\Phi}^{(3)}\left(-\frac{1}{2} \chi(z)\right)\left[\mathbb{1}-\boldsymbol{\Phi}^{(4)}\left(-\frac{1}{2} \chi(z)\right)\right]^{-1} \boldsymbol{\Phi}^{(3)} .
$$

In Eq. (47), the implicit summation over the mode indices is done without any constraints. Equation (47) and its derivation is the main result of this Letter. When transformed to the Cartesian representation, Eq. (47) becomes identical to the SCHA dynamical ansatz (Eq. (70) of Ref. [18]). We emphasize that we rigorously derived the phonon self-energy $\Pi_{r r}(z)$ using Gaussian TDVP. Our derivation theoretically proves the SCHA dynamical ansatz.

The physical interpretation of the self-energy formula we obtained vary significantly from that of the SCHA dynamical ansatz. In Gaussian TDVP, the two-phonon states are true dynamical excitations. However, in SCHA, the two-phonon
TABLE I. Excitation energy of the anharmonic oscillator [Eq. (48)] computed with three different methods.

\begin{tabular}{ll}
\hline \hline Perturbation theory & $\omega_{0}-\lambda^{2} a^{2} / 12 \omega_{0}+\mathcal{O}\left(\lambda^{4}\right)$ \\
Linearized time evolution & $\omega_{0}-\lambda^{2} a^{2} / 12 \omega_{0}+\mathcal{O}\left(\lambda^{4}\right)$ \\
Projected Hamiltonian & $\omega_{0}-\lambda^{2} a^{2} / 16 \omega_{0}+\mathcal{O}\left(\lambda^{4}\right)$
\end{tabular}

states do not have their own dynamics and appear only indirectly through the position dependence of the SCHA force constants. The presence of the dynamical two-phonon excitations is the essential reason why Gaussian TDVP can describe dynamical properties while the SCHA theory cannot.

For example, the phonon lifetime is an important dynamical property of an anharmonic lattice. In Gaussian TDVP, the one-phonon states acquire a finite lifetime by decaying to the continuum of two-phonon states through the three-phonon interaction. In contrast, in SCHA, there are no continuum states to which the one-phonon states can decay. Hence, in SCHA, the phonon lifetimes can only be described with a perturbative approximation [32] unless one resorts to an ansatz.

Discussion. A common alternative to the linearized time evolution is the projected Hamiltonian method [4,6,9]. There, the Hamiltonian is projected onto the tangent space of the variational manifold. Let us consider a single-mode anharmonic oscillator at $T=0$, whose Hamiltonian is

$\hat{H}=\frac{\omega_{0}}{2}\left(\hat{p}^{2}+\hat{r}^{2}\right)+\frac{\lambda a}{6}\left(\hat{r}^{3}-\frac{3}{2} \hat{r}\right)+\frac{\lambda^{2} b}{24}\left(\hat{r}^{4}-3 \hat{r}^{2}+\frac{3}{4}\right)$.

Here, $\lambda$ is the perturbation strength. The SCHA variational Hamiltonian is

$$
\hat{H}^{(\mathrm{H})}=\frac{\omega_{0}}{2}\left(\hat{p}^{2}+\hat{r}^{2}\right),
$$

and the variational ground-state energy is $\omega_{0} / 2$.

In Table I we list the excitation energy, the difference of the ground- and first excited-state energy, computed using different methods [38]. Comparing the variational methods to the perturbation theory, we find that the linearized time evolution is correct in the perturbative limit $\lambda \rightarrow 0$, while the projected Hamiltonian method is not. Since the SCHA dynamical ansatz is exact in the perturbative limit [18], this finding also holds for a general multimode anharmonic lattice at finite temperatures.

This difference occurs because the projected Hamiltonian method fails to describe the effect of virtual three- and fourphonon states. In Fig. 1, we show the two processes that appear in the time-domain representation of the bubble diagram for the phonon self-energy. Figure 1(b) describes a
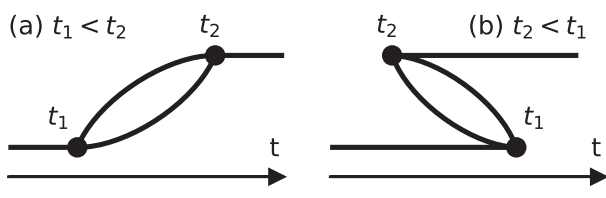

FIG. 1. Diagrams of the two processes that appear in the timedomain representation of a bubble diagram. Created using the FEYNMAN package [40]. 
process involving a four-phonon state. Since the Gaussian projected Hamiltonian method completely neglects the three- and four-phonon excitations, it only includes the process described in Fig. 1(a), not that of Fig. 1(b). In contrast, in the linearized time evolution, the coupling of the one- and two-phonon states to virtual three- and four-phonon states is included by an additional term related to the derivative of the tangent vectors, which is neglected in the projected Hamiltonian method [13]. Thanks to this additional term, the linearized time evolution gives the correct perturbative limit while the projected Hamiltonian cannot.

A promising future research direction based on our study is a rigorous, systematic expansion of the SCHA method to go beyond the harmonic approximation by using nonGaussian variational transformations [6]. Also, the use of mixed fermionic and bosonic variational states $[6,11,12]$ will allow the study of nontrivial electron-phonon correlation such as in phonon-mediated superconductivity or polarons in anharmonic lattices.

Recently, Monacelli and Mauri also reported a proof of the SCHA dynamical self-energy in an independent work [41]. While Ref. [41] additionally presents a numerical algorithm to compute the correlation functions, our work focuses on the link between TDVP and SCHA. Also, while the proof for the finite-temperature case in Ref. [41] is based on an analogy with the $T=0$ case, our proof uses purification to rigorously derive the finite-temperature equation of motion. The results of the two works are consistent when there is an overlap.

Conclusion. In summary, we developed a variational theory for the dynamical properties of anharmonic lattices using Gaussian TDVP, establishing a firm link between Gaussian TDVP and SCHA. We provided solid theoretical groundwork for the use of the SCHA dynamical ansatz in studying spectral properties. The presence of dynamical two-phonon excitations in Gaussian TDVP was essential to obtain correct dynamics of the one-phonon excitations. We compared the linearized time evolution and the projected Hamiltonian methods to find that only the former is correct in the perturbative limit. Our work establishes a useful connection between TDVP and SCHA, allowing further developments in both fields.

Acknowledgments. This work was supported by the Creative-Pioneering Research Program through Seoul National University, Korean NRF No-2020R1A2C1014760, and the Institute for Basic Science (No. IBSR009-D1).
[1] P. A. M. Dirac, Note on exchange phenomena in the Thomas atom, Math. Proc. Cambridge Philos. Soc. 26, 376 (1930).

[2] P. Kramer, A review of the time-dependent variational principle, J. Phys.: Conf. Ser. 99, 012009 (2008).

[3] J. Haegeman, J. I. Cirac, T. J. Osborne, I. Pižorn, H. Verschelde, and F. Verstraete, Time-Dependent Variational Principle for Quantum Lattices, Phys. Rev. Lett. 107, 070601 (2011).

[4] J. Haegeman, T. J. Osborne, and F. Verstraete, Post-matrix product state methods: To tangent space and beyond, Phys. Rev. B 88, 075133 (2013).

[5] Y. Ashida, T. Shi, M. C. Bañuls, J. I. Cirac, and E. Demler, Solving Quantum Impurity Problems In and Out of Equilibrium with the Variational Approach, Phys. Rev. Lett. 121, 026805 (2018).

[6] T. Shi, E. Demler, and J. Ignacio Cirac, Variational study of fermionic and bosonic systems with non-Gaussian states: Theory and applications, Ann. Phys. 390, 245 (2018).

[7] T. Guaita, L. Hackl, T. Shi, C. Hubig, E. Demler, and J. I. Cirac, Gaussian time dependent variational principle for the Bose-Hubbard model, Phys. Rev. B 100, 094529 (2019).

[8] N. Rivera, J. Flick, and P. Narang, Variational Theory of Nonrelativistic Quantum Electrodynamics, Phys. Rev. Lett. 122, 193603 (2019).

[9] L. Vanderstraeten, J. Haegeman, and F. Verstraete, Simulating excitation spectra with projected entangled-pair states, Phys. Rev. B 99, 165121 (2019).

[10] L. Vanderstraeten, J. Haegeman, and F. Verstraete, Tangentspace methods for uniform matrix product states, SciPost Phys. Lect. Notes, 7 (2019).

[11] T. Shi, E. Demler, and J. I. Cirac, Variational Approach for Many-Body Systems at Finite Temperature, Phys. Rev. Lett. 125, 180602 (2020)

[12] Y. Wang, I. Esterlis, T. Shi, J. I. Cirac, and E. Demler, Zerotemperature phases of the two-dimensional Hubbard-Holstein model: A non-Gaussian exact diagonalization study, Phys. Rev. Res. 2, 043258 (2020).

[13] L. Hackl, T. Guaita, T. Shi, J. Haegeman, E. Demler, and I. Cirac, Geometry of variational methods: Dynamics of closed quantum systems, SciPost Phys. 9, 048 (2020).

[14] D. J. Hooton, LI. A new treatment of anharmonicity in lattice thermodynamics: I, The London, Edinburgh, and Dublin Phil. Magazine and J. Sci. 46, 422 (1955).

[15] I. Errea, B. Rousseau, and A. Bergara, Anharmonic Stabilization of the High-Pressure Simple Cubic Phase of Calcium, Phys. Rev. Lett. 106, 165501 (2011).

[16] I. Errea, M. Calandra, and F. Mauri, First-Principles Theory of Anharmonicity and the Inverse Isotope Effect in Superconducting Palladium-Hydride Compounds, Phys. Rev. Lett. 111, 177002 (2013).

[17] I. Errea, M. Calandra, and F. Mauri, Anharmonic free energies and phonon dispersions from the stochastic self-consistent harmonic approximation: Application to platinum and palladium hydrides, Phys. Rev. B 89, 064302 (2014).

[18] R. Bianco, I. Errea, L. Paulatto, M. Calandra, and F. Mauri, Second-order structural phase transitions, free energy curvature, and temperature-dependent anharmonic phonons in the self-consistent harmonic approximation: Theory and stochastic implementation, Phys. Rev. B 96, 014111 (2017).

[19] L. Monacelli, I. Errea, M. Calandra, and F. Mauri, Pressure and stress tensor of complex anharmonic crystals within the stochastic self-consistent harmonic approximation, Phys. Rev. B 98, 024106 (2018).

[20] R. Bianco, I. Errea, M. Calandra, and F. Mauri, High-pressure phase diagram of hydrogen and deuterium sulfides from first principles: Structural and vibrational properties including quantum and anharmonic effects, Phys. Rev. B 97, 214101 (2018).

[21] U. Aseginolaza, R. Bianco, L. Monacelli, L. Paulatto, M. Calandra, F. Mauri, A. Bergara, and I. Errea, Phonon Collapse 
and Second-Order Phase Transition in Thermoelectric SnSe, Phys. Rev. Lett. 122, 075901 (2019).

[22] I. Errea, M. Calandra, C. J. Pickard, J. Nelson, R. J. Needs, Y. Li, H. Liu, Y. Zhang, Y. Ma, and F. Mauri, High-Pressure Hydrogen Sulfide from First Principles: A Strongly Anharmonic Phonon-Mediated Superconductor, Phys. Rev. Lett. 114, 157004 (2015).

[23] I. Errea, M. Calandra, C. J. Pickard, J. R. Nelson, R. J. Needs, Y. Li, H. Liu, Y. Zhang, Y. Ma, and F. Mauri, Quantum hydrogenbond symmetrization in the superconducting hydrogen sulfide system, Nature (London) 532, 81 (2016).

[24] M. Borinaga, U. Aseginolaza, I. Errea, M. Calandra, F. Mauri, and A. Bergara, Anharmonicity and the isotope effect in superconducting lithium at high pressures: A first-principles approach, Phys. Rev. B 96, 184505 (2017).

[25] I. Errea, F. Belli, L. Monacelli, A. Sanna, T. Koretsune, T. Tadano, R. Bianco, M. Calandra, R. Arita, F. Mauri, and J. A. Flores-Livas, Quantum crystal structure in the 250-kelvin superconducting lanthanum hydride, Nature (London) 578, 66 (2020).

[26] M. Leroux, I. Errea, M. Le Tacon, S.-M. Souliou, G. Garbarino, L. Cario, A. Bosak, F. Mauri, M. Calandra, and P. Rodière, Strong anharmonicity induces quantum melting of charge density wave in $2 \mathrm{H}-\mathrm{NbSe}_{2}$ under pressure, Phys. Rev. B 92, 140303(R) (2015).

[27] R. Bianco, I. Errea, L. Monacelli, M. Calandra, and F. Mauri, Quantum Enhancement of Charge Density Wave in $\mathrm{NbS}_{2}$ in the Two-Dimensional Limit, Nano Lett. 19, 3098 (2019).

[28] J. S. Zhou, L. Monacelli, R. Bianco, I. Errea, F. Mauri, and M. Calandra, Anharmonicity and Doping Melt the Charge Density Wave in Single-Layer TiSe ${ }_{2}$, Nano Lett. 20, 4809 (2020).

[29] R. Bianco, L. Monacelli, M. Calandra, F. Mauri, and I. Errea, Weak Dimensionality Dependence and Dominant Role of Ionic Fluctuations in the Charge-Density-Wave Transition of $\mathrm{NbSe}_{2}$, Phys. Rev. Lett. 125, 106101 (2020).

[30] J. Sky Zhou, R. Bianco, L. Monacelli, I. Errea, F. Mauri, and M. Calandra, Theory of the thickness dependence of the charge density wave transition in 1T-TiTe 2 , 2D Mater. 7, 045032 (2020).
[31] J. Diego, A. H. Said, S. K. Mahatha, R. Bianco, L. Monacelli, M. Calandra, F. Mauri, K. Rossnagel, I. Errea, and S. BlancoCanosa, Van der Waals driven anharmonic melting of the 3D charge density wave in $\mathrm{VSe}_{2}$, Nat. Commun. 12, 598 (2021).

[32] L. Paulatto, I. Errea, M. Calandra, and F. Mauri, First-principles calculations of phonon frequencies, lifetimes, and spectral functions from weak to strong anharmonicity: The example of palladium hydrides, Phys. Rev. B 91, 054304 (2015).

[33] U. Aseginolaza, R. Bianco, L. Monacelli, L. Paulatto, M. Calandra, F. Mauri, A. Bergara, and I. Errea, Strong anharmonicity and high thermoelectric efficiency in high-temperature SnS from first principles, Phys. Rev. B 100, 214307 (2019).

[34] U. Aseginolaza, T. Cea, R. Bianco, L. Monacelli, M. Calandra, A. Bergara, F. Mauri, and I. Errea, Bending rigidity and sound propagation in graphene, arXiv:2005.12047.

[35] L. Monacelli, I. Errea, M. Calandra, and F. Mauri, Black metal hydrogen above $360 \mathrm{GPa}$ driven by proton quantum fluctuations, Nat. Phys. 17, 63 (2021).

[36] C. Weedbrook, S. Pirandola, R. García-Patrón, N. J. Cerf, T. C. Ralph, J. H. Shapiro, and S. Lloyd, Gaussian quantum information, Rev. Mod. Phys. 84, 621 (2012).

[37] G. Adesso, S. Ragy, and A. R. Lee, Continuous variable quantum information: Gaussian states and beyond, Open Syst. Inf. Dynam. 21, 1440001 (2014).

[38] See Supplemental Material at http://link.aps.org/supplemental/ 10.1103/PhysRevResearch.3.L032017 for the analysis of the variational parameters, technical details of the derivations, a note on degeneracies, a note on the zero-temperature case, and the calculation of the excitation energy of the single-mode anharmonic Hamiltonian, and it includes Ref. [42].

[39] M. A. Nielsen and I. L. Chuang, Quantum Computation and Quantum Information (Cambridge University Press, Cambridge, England, 2000).

[40] http://gkantonius.github.io/feynman/, accessed: 2020-05-31.

[41] L. Monacelli and F. Mauri, Time-dependent self-consistent harmonic approximation: Anharmonic nuclear quantum dynamics and time correlation functions, Phys. Rev. B 103, 104305 (2021).

[42] R. Pathria and P. D. Beale, Statistical Mechanics, 3rd ed. (Academic Press, Boston, MA, 2011). 